\title{
Participación de pescadores artesanales en el diseño de un proyecto de implantación de arrecifes artifi- ciales en el Sur de Brasil
}

\author{
Participation of Small-Scale Fishermen in the Design of a Project \\ for Creating Artificial Reefs in the South of Brazil
}

\author{
JOSÉ MILTON ANDRIGUETTO-FILHO \\ Universidad Federal de Paraná, Brasil \\ NAÍNA PIERRI \\ Universidad Federal de Paraná, Brasil
}

Resumen Desde 2004, está en curso, en el litoral del estado de Paraná (Brasil), un proyecto para lanzamiento de arrecifes artificiales y unidades antiarrastre en la plataforma costera rasa. Éste pretende restringir la pesca de arrastre de fondo de gran tamaño para proteger y recuperar la biodiversidad marina y los stocks pesqueros, y así favorecer la sustentabilidad de la pesca de pequeña escala. En 2005 , los autores fueron invitados para conducir un proceso de participación pública de los pescadores directamente afectados. La metodología consistió en organizar cuatro fórums municipales de discusión, más una reunión final conjunta. Cada instancia fue precedida de una intensa divulgación y se usaron diversas técnicas de comunicación y participación. A partir de una propuesta inicial de disposición de las estructuras, y de la presentación de las ventajas y desventajas de la intervención, el primer objetivo fue permitir a los pescadores aceptar o no la propuesta. Habiendo aceptación, se trató de determinar los detalles de la distribución espacial de las estructuras. La aceptación fue amplia, a excepción del municipio en que se practica la pesca de arrastre de escala industrial. El proceso reveló conflic- 
tos internos en la sociedad pesquera, así como lagunas en el conocimiento de los investigadores sobre la pesca y la ecología local, y sobre los posibles impactos del proyecto, lo que motivó su rediseño. Esta consulta constituyó un proceso democrático y participativo sin precedentes en la historia de la gestión pesquera en Paraná, que pasó a ser referencia regional y nacional.

PALABRAS CLAVE Gestión de pesca, participación pública, arrecifes artificiales, pescadores artesanales.

ABSTRACT Since 2004, a project has been in progress off the coast of Paraná State (Brazil) to set up artificial reefs and anti-trawling units on the shallow continental shelf. The object is to restrict large-scale bottom trawling in order to protect and recover marine biodiversity and fish stocks, thus favouring the sustainability of small-scale fishing. In 2005 , the authors were invited to carry out a public participation process for the fishermen directly affected. The method consisted in organising four municipal discussion forums, plus a final joint meeting. Each event was preceded by intense publicity, and various communication and information techniques were used. Starting from an initial proposal for the location of the structures, and a presentation on the advantages and disadvantages of intervention, the first objective was to allow the fishermen to accept or reject the proposal. If it was accepted, the next decision was to fix the details of the spatial distribution of the structures. Acceptance was widespread, except in one district where industrial-scale trawling is practiced. The process revealed internal conflicts in the fishermen's society, as well as gaps in the researchers' knowledge of fisheries and the local ecology, and the possible impacts of the project, leading to redesign. This consultation constituted an unprecedented democratic, participative process in the history of fishery management in Paraná, which has become a regional and national reference.

KEYWORDS Fishery management, public information, artificial reefs, smallscale fishermen.

\section{Introducción}

El litoral del Estado de Paraná se localiza entre los paralelos $25^{\circ}$ y $26^{\circ}$ de latitud sur y $48^{\circ}$ y $49^{\circ}$ de longitud oeste. Posee una superficie total de $6.600 \mathrm{~km}$ cuadrados, y $98 \mathrm{~km}$ de extensión (figura I). La planicie costera es caracteriza- 
da por extensas playas arenosas expuestas, separadas por las bahías de Guaratuba y Paranaguá (Bigarella y otros, I978). Los ambientes marinos abarcan un área aproximada de $3.000 \mathrm{~km}^{2}$ considerando el límite de I 2 millas del mar territorial brasilero, y comprenden ecosistemas de manglares, restingas, costas rocosas y playas, entre otros, con numerosos recursos de interés ecológico y económico (Sema, 2006).

Desde el punto de vista administrativo, el litoral del Paraná es constituido por siete municipios: Guaraqueçaba, Antonina, Morretes, Paranaguá, Pontal do Paraná, Matinhos y Guaratuba, siendo costeros con excepción de Morretes. Según medición de 2007 , posee una población total de 245.845 personas, en su gran mayoría residentes en áreas urbanas (IBGE, 2007).

En este litoral hay 60 villas de pescadores con 5 o más domicilios. Se distribuyen dentro y fuera de las bahías, en ambientes urbanos y rurales (Andriguetto Filho, I999). En 2003, había un total de 4.277 pescadores profesionales registrados en la Secretaria Especial de Pesca (SEAP), que disponían de una flota de 930 embarcaciones registradas en el Instituto Brasilero de Medio Ambiente (IBAMA) (Andriguetto-Filho y otros, 2006a). Considerando el grupo familiar de los pescadores, se estima que unas I 2 a I 5.000 personas del litoral dependen o están siendo beneficiadas por esta actividad. La distribución espacial de los pescadores en los 6 municipios costeros es bastante desigual: la mitad se concentra entre Guaraqueçaba y Paranaguá, en partes aproximadamente iguales; Guaratuba, concentra 2r \%; Antonina, I 6,5\%; Pontal, 8,6\%; y Matiños, 5\% (Pierri y otros, 2006).

El litoral del Paraná presenta una gran diversidad de modalidades y prácticas de pesca. Se trata, fundamentalmente, de pesca de pequeña escala o «artesanal». Sin embargo, embarcaciones industriales locales y procedentes de otros Estados también operan en la costa paranaense. La producción es relativamente baja, comparada a los demás Estados de la región sur de Brasil (Vasconcellos y otros, 2005; Andriguetto-Filho y otros, 2006a).

La pesca más importante, en volumen, valor económico y cantidad de pescadores involucrados, es la que tiene como objetivo el camarón blanco (Litopenaeusschimitti) y el camarón siete-barbas (Xiphopenaeuskroyeri), siendo responsable por el $73 \%$ de los desembarques totales, en términos de peso ( Natividade y otros, 2004). La captura de camarón es realizada de diferentes formas, siendo la más practicada la pesca de arrastre de fondo, que es la única de carácter industrial presente en la región (Andriguetto-Filho, 1999). Este tipo de arrastre es considerado una de las prácticas pesqueras más problemáticas, 
pues junto con la especie-objetivo son capturados y descartados juveniles y larvas de otras especies afectando el ecosistema, la biodiversidad y, por tanto, la productividad pesquera. Según Funpar (2005), en la región sur brasileña, ese descarte llega a cuatro kilos para cada kilo de camarón capturado.

Estudios sobre el estado de los stocks de recursos pesqueros en la región sur de Brasil evidenciaron la existencia de una situación comprometida y de evidente sobrepesca (Dias-Neto y Dornelles, I996). Sumado a eso, conflictos presentes en los sistemas de producción pesquera ponen en riesgo la sustentabilidad de la pesca y la sobrevivencia de las comunidades de pescadores artesanales, cuya disolución implicaría importantes costos económicos, sociales y culturales. Según Andriguetto-Filho (1999), hay conflictos internos derivados del acceso libre a los recursos y de la competencia entre distintas escalas y modalidades de pesca. Es lo que se observa, por ejemplo, entre los pescadores paranaenses de pequeña escala y los grandes barcos arrastreros de otros Estados;

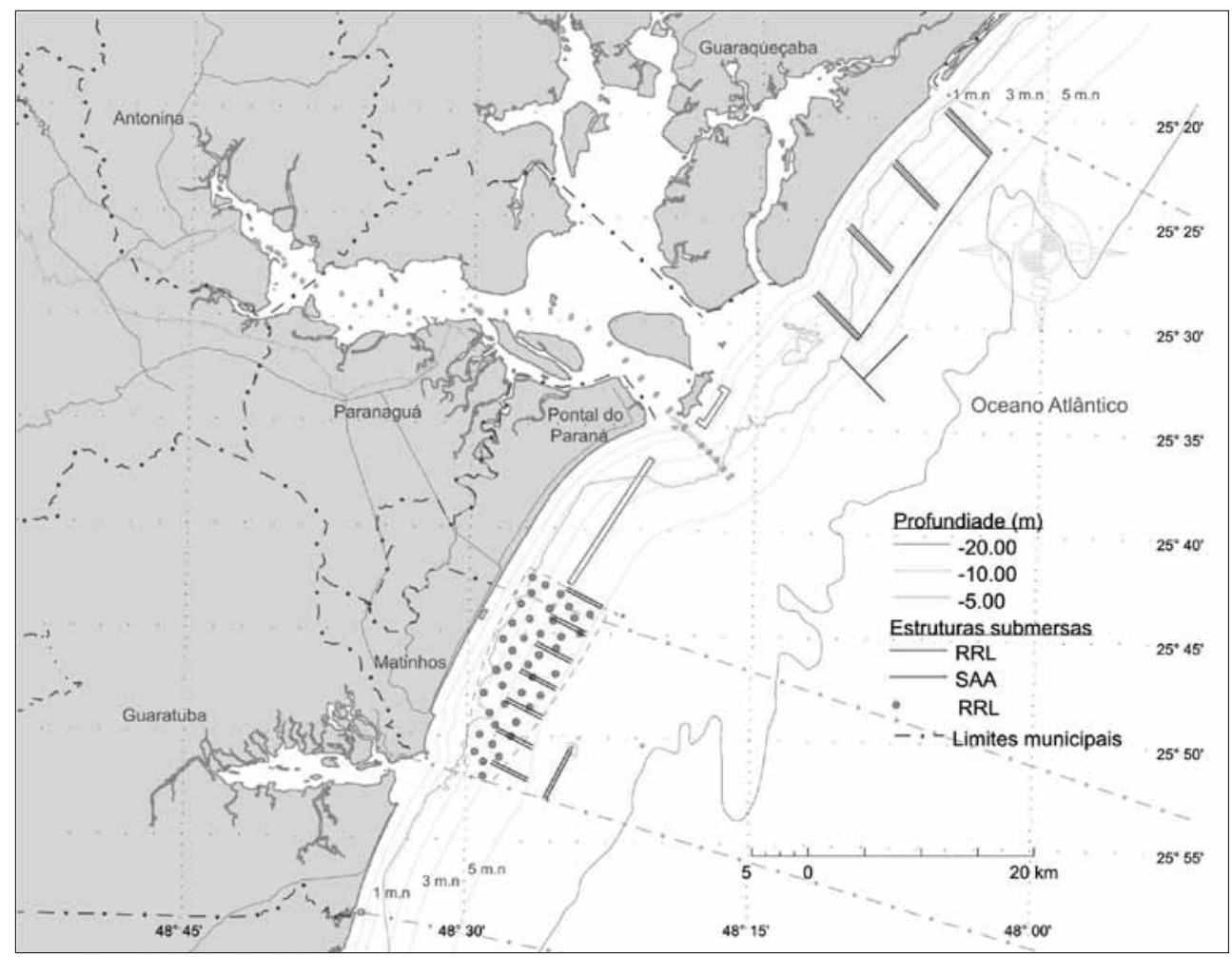

Figura 1. Litoral del Estado de Paraná, con la distribución de los arrecifes artificiales determinada por los pescadores tras el final del proceso de consulta. Se enumeran las ciudades y accidentes geográficos mencionados en el texto, así como las distancias de la costa en millas náuticas ( $\mathrm{mn}$ en la parte superior de la figura) y la profundidad en metros (parte inferior). 
y en el contexto local, entre los arrastreros de camarón con embarcaciones de tamaño medio y los pescadores que practican pesca de enmalle de deriva (camarones y peces) y enmalle fijo (peces).

Las medidas de gestión pesquera actualmente en vigor han sido poco eficaces en relación a la recuperación de los stocks de recursos y la resolución de los conflictos presentes. Así, la implantación de arrecifes artificiales parece una alternativa interesante porque, sin necesidad de fiscalización y de forma permanente, contribuiría a la recuperación de la biodiversidad marina, creando hábitats favorables al reclutamiento de larvas y protegiendo la presencia de diversas especies. Dentro de esa propuesta general, implantar estructuras antiarrastre, que son arrecifes artificiales especialmente diseñados para retener las redes de arrastre de fondo, inhibiría esta modalidad de pesca, que es la más depredadora en la región. Por otro lado, los arrecifes artificiales crean condiciones favorables para desarrollar maricultura, que, se bien planeada y realizada, es una opción interesante tanto desde el punto de vista ecológico como económico.

En el litoral del Paraná, el lanzamiento de arrecifes artificiales y unidades antiarrastre se inició con un proyecto de carácter experimental, bajo responsabilidad de investigadores de la Universidad Federal de Paraná (UFPR) y del instituto Ecoplan (una ONG), denominado RAM, Recifes Artificiais Marinhos (arrecifes artificiales marinos), que fue ejecutado entre los años I997 y 2002, instalándose más de 2000 estructuras en la plataforma interna, de diferentes tipos y modelos, incluyendo unidades antiarrastre (Brandini, 2003). Según los responsables, los resultados obtenidos demostraron la necesidad de dar continuidad a la «creación de áreas de exclusión de pesca de arrastre y de nuevos núcleos de biodiversidad». Por eso fue elaborado el proyecto Rebimar, «Recuperación de la biodiversidad marina» y de los stocks pesqueros tradicionales del litoral del Paraná a través de un sistema antiarrastre bajo responsabilidad de los mismos investigadores de la UFPR involucrados en el proyecto anterior. En su versión original del año 2005 (Funpar, 2005), el proyecto se proponía instalar 3.840 unidades antiarrastre y 6.000 bloques de arrecifes, abarcando toda la plataforma rasa de Paraná (Santos, 2007). Así, la diferencia fundamental de este proyecto con el anterior es la escala, significativamente mayor, de donde sus impactos ambientales y sociales, serían, correspondientemente, mayores.

La extensión del territorio donde serían implantados y la cantidad de estructuras previstas podrían alterar significativamente las condiciones de la pes- 
ca de mar abierto, con previsión de impactos positivos y negativos directos para la población de pescadores que depende de esa actividad, e indirectos para toda la economía regional. Eso justificó el proceso de consulta realizado en 2006, especialmente dirigido a los pescadores profesionales de mar abierto, conducido paralelamente al proyecto de lanzamiento de arrecifes propiamente dicho, y por un equipo distinto al de éste (Andriguetto-Filho y otros, 2006b).

Desde el punto de vista económico-social, los arrecifes artificiales beneficiarían potencialmente a todos los pescadores, por el aumento de biodiversidad, mayor disponibilidad de recursos pesqueros y oportunidades de diversificación de ingresos a través de actividades como la pesca turística o cultivos marinos asociados. Ya la implantación de las unidades antiarrastre podría excluir determinadas áreas de la actividad de arrastre de fondo con puertas, además de proteger los propios arrecifes artificiales, protegiendo la biodiversidad en algunas partes de la plataforma interna. Esta limitación de las áreas de pesca teóricamente se aplicaría más a las embarcaciones arrastreras de gran tamaño (segmentos industriales, principalmente de otros Estados, pero también locales), y favorecería a las embarcaciones arrastreras menores (segmentos artesanales paranaenses), y también a los que utilizan otras artes de pesca.

La comprensión de que la implantación de esas estructuras puede implicar beneficios y perjuicios diferentes a los diferentes sectores de pescadores, al planificar la consulta pública, sumó a la exigencia de convocar a la mayor cantidad posible de pescadores, la exigencia de que los diferentes grupos de interés estuviesen representados. Así el desafío fue montar un proceso que facilitase la explicitación de los distintos intereses, ofreciese un camino para llegar a los consensos posibles y necesarios, y se arribase a una propuesta común de los pescadores para todo el litoral del Paraná.

\section{Materiales y métodos}

La metodología contó con dos grandes componentes: I) un conjunto de reuniones o foros públicos; 2) un esfuerzo intenso y estructurado de divulgación y preparación. Se trataba de obtener la divulgación más amplia posible del proyecto, aportar informaciones suficientes para el proceso de toma de decisiones, y maximizar la oportunidad de participación, voz y voto de cada pescador. Deliberadamente, el proceso no fue dirigido a otros grupos de interés, con autorización de la agencia ambiental, pues sólo el sector pesquero podría sufrir eventuales impactos negativos del proyecto, además de que una parte de éste, 
el subsector artesanal, está protegido por la legislación brasileña en calidad de población tradicional.

El equipo de investigadores que condujo el proceso de consulta contó con I 5 participantes, en su mayoría profesionales graduados, tanto de las ciencias sociales como de las naturales. Los miembros de mayor calificación tenían, por lo menos, I 5 años de experiencia e intimidad con la problemática concreta de la pesca en la región objetivo del proyecto. El equipo recibió capacitación específica en tres talleres durante la primera semana de trabajo, más un taller en la víspera de cada fórum.

El proceso de participación, que se desarrolló entre mayo y octubre de 2006, se organizó en torno de cuatro grandes reuniones públicas, una para cada municipio en que están presentes los sistemas pesqueros afectados por el proyecto, a saber, Guaratuba, Matinhos, Pontal do Paraná y Guaraqueçaba. Culminó con una reunión técnica final de representantes, con presencia de observadores institucionales, en el municipio de Pontal do Paraná. Dada su pequeña frente oceánica, limitada a la Ilha do Mel, y el pequeño contingente de pescadores potencialmente afectados por el proyecto, no se realizó una reunión específica para el municipio de Paranaguá, siendo sus pescadores incluidos en la reunión de Pontal do Paraná, y llamados también para las reuniones de Guaratuba y Guaraqueçaba. Los procedimientos adoptados permitieron abarcar todos los tipos de flota, toda el área de actuación de las flotas de arrastre, y, potencialmente, toda la población pesquera que trabaja en mar abierto en Paraná.

La estrategia consistió en aproximaciones sucesivas al público-objetivo, cada vez más detalladas y socializadas. Para cada municipio, se elaboraron previamente listas de contactos (nombres, teléfonos y direcciones), lo que fue facilitado por la experiencia del equipo. Fueron seleccionados pescadores que tuviesen un perfil de liderazgo o capacidad de divulgación y formación de opinión. Fueron siempre incluidos los líderes formales, especialmente los presidentes de las colonias de pesca, pero también se tuvo cuidado de considerar la presencia de líderes políticos, religiosos o de otro tipo, convocando representantes de todas partes. Las etapas del proceso para cada municipio fueron las siguientes:

I. Contacto telefónico previo, con tantos nombres de la lista cuanto fuera posible, con el objetivo de anunciar el comienzo de los trabajos en el municipio y definir la fecha de la próxima conversación.

2. Al día siguiente, o a pocos días después de la etapa anterior, se realiza- 
ron la primera visita al municipio y contactos personales individuales con los líderes y formadores de opinión, con los objetivos de presentar la propuesta del proyecto en líneas generales y de preparar la etapa siguiente. El contacto no se restringió a los nombres previamente listados, si no que se siguió la técnica de la «bola de nieve», solicitando a cada contacto que indicase nuevos nombres. Los contactos fueron hechos por un par de miembros del equipo, idealmente un hombre y una mujer (para facilitar la aproximación a las mujeres), siendo el número de parejas simultáneamente en campo nunca inferior a tres. En esta etapa, se tomó especial cuidado en visitar todas las villas, barrios o localidades del municipio que pudiesen tener pescadores de mar abierto, buscándose, en cada caso, un responsable para la divulgación de la próxima etapa. También se buscó el contacto y apoyo del gobierno municipal para la divulgación y logística.

3. La reunión de líderes y formadores de opinión era realizada en el día establecido en la etapa anterior (usualmente uno o dos días después). En esta reunión participaban los pescadores contactados anteriormente, así como sus invitados. La reunión era usualmente corta (una a dos horas), en forma de mesa redonda. La propuesta original del proyecto, preparada por los investigadores, era presentada detalladamente a los presentes, se aclaraban dudas y se anotaban las opiniones de los pescadores. Copias de fotos de los arrecifes y del mapa con la disposición originalmente prevista eran distribuidas y dejadas a los presentes, pidiéndose que promovieran la discusión con la comunidad antes de la realización de la reunión municipal. El local, fecha y horario para la realización de la reunión pública eran definidos por los propios pescadores, según su conveniencia. Otros objetivos eran:

- mostrar que el proceso de participación pública permitiría que los pescadores, de hecho, interfiriesen profundamente en el resultado final (en función de la disposición espacial de los arrecifes), inclusive rechazando el lanzamiento de toda estructura;

- explicar las posibilidades del diseño de los campos de arrecifes;

- explicar cómo funcionaría la reunión pública (para que los pescadores percibieran cómo tendrían que trabajar en grupo y eventualmente encarar sus diferencias internas hasta llegar a una propuesta de mapa de distribución de las instalaciones deseadas); y 
- explicitar que el tiempo hasta la realización del fórum serviría para que distribuyeran la noticia y discutieran entre sí.

4. En el periodo entre la reunión de líderes y la reunión pública municipal, el equipo promovió la divulgación del evento en dos frentes: i) por los medios de comunicación locales, en radios y diarios, y ii) por un trabajo cara a cara, con retorno a las mismas localidades visitadas en la etapa 2, para colocación de carteles, distribución de materiales impresos y refuerzo verbal, inclusive a nuevos contactos. Los carteles fueron colocados en locales específicamente seleccionados en función de la frecuencia de presencia de pescadores, como las sedes de las colonias de pesca, mercados y empresas de pescado.

5. Las reuniones públicas, que son el corazón del proceso, serán descritas en detalle más adelante. Fueron realizadas una o dos semanas después de la reunión de líderes, tiempo suficientemente largo para permitir la asimilación del proceso por los pescadores, pero no tan largo de modo que supusiera un enfriamiento de la motivación creada por las fases anteriores.

Las reuniones duraban cerca de cuatro horas y seguían una programación definida, con pequeñas adaptaciones al caso de cada municipio (Tabla I).

La presentación de apertura, corta y objetiva, tenía diversos objetivos importantes: explicaba la programación y objetivos de la reunión, presentaba la historia del proyecto y las posibilidades de disposición espacial de las estructuras (a ser diseñada por los pescadores), buscaba dejar claros los posibles costos y beneficios del proyecto para los pescadores, y explicitaba que la no aceptación integral del proyecto era una opción tan válida como cualquier propuesta de disposición de los arrecifes.

Los pescadores se dividían en grupos de trabajo, cuidando que su composición fuese heterogénea en términos de intereses, para que la negociación necesaria ocurriese en la elaboración de la propuesta. Estos grupos de trabajo eran siempre acompañados por una pareja de monitores, siendo usualmente un miembro senior y un junior del equipo. Entre las funciones de los monitores estaban: i) aclarar las dudas de los participantes, ii) controlar el tiempo, iii) mediar posibles conflictos, iv) tomar notas y elaborar la descripción textual de la propuesta del grupo (marcas, isobatas, distancias, detalles constructivos, etc.) y, de modo general, v) buscar orientar a los participantes para que se alcanzasen los objetivos. Los monitores fueron explícitamente instruidos a no asumir 
el trabajo del grupo, a no dar sugerencias ellos mismos y a dejar claro que la propuesta podía consistir en la ausencia total de las estructuras, reforzando lo que ya había sido dicho en la presentación de apertura. La focalización y motivación inicial del grupo eran conseguidas pidiéndose a los participantes que, por cerca de ro minutos, discutiesen en parejas o tríos, sin la participación de los monitores, las siguientes preguntas:

- ¿Qué puede ganar o perder usted con el proyecto?

- ¿Eso vale para todos los que hacen el mismo tipo de pesca que usted?

- ¿Cómo usted podría cambiar el proyecto para sacar mejor provecho? (O sea, responder al desafío propuesto en la presentación de apertura).

El procedimiento para la elaboración de la propuesta de cada subgrupo consistía en diseñar la disposición de las estructuras en papel vegetal, sobrepuesto a una carta náutica de base. Los pescadores podían definir tipo, número y posición de los arrecifes artificiales y unidades antiarrastre. Además de material de dibujo, estaban a su disposición reglas con marcas en millas náuticas en la escala de la carta, y trozos de palitos de madera para simular los espigones de arrecifes propuestos en el proyecto original, también en escala. Se enfatizaba que toda el área de actuación de la flota municipal podría ser mapeada. El objetivo era generar un mapa con la sugerencia de distribución de los arrecifes a lo largo del área, representando una propuesta única acordada por el grupo. Durante el trabajo, los monitores procuraban identificar los padrones en las ideas individuales y explicitarlos para el grupo, así como procuraban crear espacios para que aun los más tímidos pudiesen expresarse.

En todo el proceso, el lenguaje y los medios de trabajo empleados por el equipo consideraron explícitamente el contexto cultural y lingüístico del público-objetivo. Éste fue un aspecto ampliamente favorecido por la experiencia del equipo. En particular, fue tomado en cuenta el analfabetismo funcional de algunos pescadores. El llenado de las fichas de registro, por ejemplo, era siempre hecho por un miembro del equipo, para evitar situaciones embarazosas. De la misma forma, en la presentación de apertura, todo lo que aparecía por escrito en la pantalla era verbalizado por el presentador.

Inmediatamente después de cada reunión, cada participante recibía una copia del resultado, con un mapa preliminar y una descripción textual de la propuesta recién aprobada. Este documento se constituía en un elemento de transparencia y garantía de la honestidad del proceso para sus participantes, 
Tabla 1. Programación de una reunión pública de participación de los pescadores en el diseño del proyecto de arrecifes artificiales en el litoral del Paraná

\begin{tabular}{lll} 
Etapa & $\begin{array}{l}\text { Horario o } \\
\text { duración }\end{array}$ & Descripción y objetivos \\
\hline $\begin{array}{l}\text { Organización del espacio } \\
\text { (ambiente y equipamientos) } \\
\text { Preparación de la merienda }\end{array}$ & $\begin{array}{l}\text { Comienzo al } \\
\text { menos dos } \\
\text { horas antes } \\
\text { de la reunión }\end{array}$ & $\begin{array}{l}\text {. Arreglo de las sillas y mesas; iluminación; instalación de los } \\
\text { equipos de informática y audiovisuales, etc., previéndose los } \\
\text { momentos de plenaria y los de grupos de trabajo. }\end{array}$ \\
\hline Filmación y fotografía & $\begin{array}{l}\text { Durante todo galletitas disponibles desde el comienzo. } \\
\text { el evento }\end{array}$ & \\
\hline
\end{tabular}

Registro de los participantes y recogimiento de firmasen el libro de asistencia

\section{Comienzo una} hora antes de la reunión
- Llenar las fichas de registro (una por participante). Se debían obtener los datos personales habituales, e informaciones sociales y profesionales para orientar la conducción de los trabajos de grupo y permitir la interpretación posterior de los resultados.

- Entrega de la carpeta y Manual del Participante. Éste trae orientación sobre los objetivos y funcionamiento de la reunión, y sugerencias de participación.

- Apertura, con lectura del término oficial.

Cerca de 20

- Presentación del equipo y eventuales observadores.

- Presentación en multimedia. minutos

Grupos de trabajo para la elaboración de las propues- 1:20 tas parciales (GTs)

Ver detalle a continuación.

$\begin{array}{ll}\text { Intervalo con merienda } & \begin{array}{l}\text { Cerca de } 20 \\ \text { minutos }\end{array}\end{array}$

Síntesis de las propuestas por el equipo del proyecto

Cerca de 20 minutos
- Simultáneamente a la merienda, los mapas y las propuestas elaboradas por cada grupo fueron sintetizados por el equipo. - Se preparaba una propuesta única para ser perfeccionada y validada en la etapa siguiente, y se identificaban elementos individuales a ser discutidos y votados.

\begin{tabular}{|c|c|c|}
\hline $\begin{array}{l}\text { Elaboración de la propuesta } \\
\text { final }\end{array}$ & 1:30 & $\begin{array}{l}\text { - Nuevamente en asamblea general, presentación de las pro- } \\
\text { puestas individuales de cada grupo por uno de sus miembros, } \\
\text { seguida de la presentación de la síntesis, y cómo se llegó a ella, } \\
\text { por el equipo. } \\
\text { - Discusión, corrección y perfeccionamiento de la propuesta de } \\
\text { síntesis por los pescadores. } \\
\text { - Votación de la propuesta global y/o de componentes } \\
\text { individuales. Fueron mantenidos en la propuesta del municipio } \\
\text { apenas los componentes con votación mayoritaria. }\end{array}$ \\
\hline Finalización de la reunión & $\begin{array}{l}\text { Cerca de } 30 \\
\text { minutos }\end{array}$ & $\begin{array}{l}\text { - Minipresentación recordando los encaminamientos } \\
\text { siguientes y la necesidad de apropiación y acompañamiento } \\
\text { del proceso por los pescadores. } \\
\text { - Definición de los representantes para defender la propuesta } \\
\text { municipal en la reunión final. } \\
\text { - Impresión y entrega a los participantes de la propuesta } \\
\text { aprobada (mapas y texto). }\end{array}$ \\
\hline
\end{tabular}


que con frecuencia se mostraron incrédulos de que sus propuestas serían debidamente consideradas en etapas posteriores.

Después de las reuniones municipales, se realizó la reunión pública final, con los objetivos de crear un foro de desempate de posibles incompatibilidades entre las propuestas municipales, y de servir como una instancia formal de observación para representantes institucionales. Aunque la reunión haya sido pública y abierta, fueron expresamente convidados delegados de las instituciones con responsabilidades técnicas o administrativas sobre el proyecto, además, obviamente, de los representantes de los pescadores elegidos en las reuniones municipales. La premisa política básica para esta reunión fue la de que las reuniones municipales satisfacieran las condiciones necesarias y suficientes para el componente participativo y democrático del proceso. Así, para que no se ofendiese la soberanía de la elección popular hecha en las reuniones anteriores, en esta quinta reunión no cabría proponer el aumento de estructuras, mientras la retirada sólo podría ser propuesta en aquellos casos en que diseños diferentes y conflictivos hubieran sido propuestos por las reuniones municipales para un mismo trecho de la costa.

En consonancia, esta reunión tuvo formato diferente a las anteriores, comenzando por la presentación de los participantes, del objetivo y programación de la reunión, del proyecto original y del propio proceso participativo conducido hasta entonces. El núcleo de la reunión fue la presentación de las propuestas municipales por los propios pescadores y de la síntesis producida por el equipo técnico, seguida de la discusión de los casos particulares, con las opciones de mantener o eliminar elementos del diseño final. La reunión terminó con la elección de representantes de los pescadores presentes para componer, junto con los responsables técnicos del proyecto, una comisión técnica permanente, para definir los detalles finales del proyecto (como la forma de señalar o marcar las estructuras para navegación) y garantizar el acompañamiento de su ejecución y el lanzamiento de las estructuras al mar.

\section{Resultados y discusión}

En las etapas previas a las reuniones municipales, se visitaron en total 33 villas o barrios pesqueros en los cinco municipios con pesca en mar abierto. Un total de 7 I pescadores participó de las reuniones de líderes, mientras I 92 participaron de las reuniones municipales. La tabla 2 muestra el número de pescadores por reunión y municipio. En lo que se refiere a la composición de los partici- 
Tabla 2. Características de las reuniones públicas de participación de los pescadores en el diseño del proyecto de implantación de arrecifes artificiales en el litoral del Paraná

\begin{tabular}{|c|c|c|c|c|c|c|}
\hline Municipio & $\begin{array}{l}\text { Fecha } \\
(2006)\end{array}$ & Local & Duración & Participantes & $\begin{array}{l}\text { Pescadores/ } \\
\text { Municipio }\end{array}$ & $\begin{array}{c}\text { Porcentaje } \\
\text { participantes }\end{array}$ \\
\hline Matinhos & $07 / 06$ & $\begin{array}{l}\text { Mercado Muni- } \\
\text { cipal de Pescado }\end{array}$ & $16: 00$ a $22: 00$ & 57 & 215 & 26,5 \\
\hline Pontal do Paraná & 07/07 & $\begin{array}{l}\text { Casa da Cultura } \\
\text { de Ipanema }\end{array}$ & $16: 00$ a $20: 00$ & 37 & 365 & 10,1 \\
\hline Guaratuba & $29 / 07$ & $\begin{array}{l}\text { Secretaria } \\
\text { Municipal de } \\
\text { Educação }\end{array}$ & $17: 00$ a 21:00 & $\begin{array}{c}56 \\
\text { (siendo } 32 \text { pes- } \\
\text { cadores de mar } \\
\text { abierto) }\end{array}$ & $\begin{array}{c}\sim 660 \text { de } \\
\text { mar abierto }\end{array}$ & 4,8 \\
\hline Guaraqueçaba & 03/09 & $\begin{array}{l}\text { Esporte Clube } \\
\text { Guaraqueçaba }\end{array}$ & $11: 00$ a $16: 30$ & 28 & $\begin{array}{l}\text { 300 (en } \\
\text { las } 3 \text { villas } \\
\text { abarcadas) }\end{array}$ & 9,3 \\
\hline
\end{tabular}

pantes, las diversidades relevantes (lugar de origen, tipos, locales y recursos de pesca, nivel tecnológico y económico) estuvieron representadas, lo que se puede constatar por la lista de asistentes y por la base de datos elaborada a partir de las fichas de inscripción.

\section{Lecciones aprendidas: las dimensiones técnica y ecológica}

La aceptación de la intervención propuesta fue amplia, tanto que el número de estructuras determinado por los pescadores excedió sensiblemente la capacidad del proyecto (figura I). Las propuestas siguieron una norma tradicional de manejo entre los pescadores, que es la división espacial del territorio, lo que no llegó a ser una sorpresa. Concretamente, cada municipio propuso estructuras sólo para su frente oceánico. Las pocas excepciones (seis situaciones) fueron dirimidas por acuerdo durante la reunión final, siendo tres aprobadas y tres rechazadas por los representantes de los municipios afectados.

La magnitud de las propuestas pareció correlacionada al nivel técnico-económico de la pesca en cada municipio. Así, solamente en la reunión del municipio de Guaratuba hubo un rechazo general a la instalación de los arrecifes artificiales. Este municipio abriga el sistema pesquero más próximo a la pesca industrial en el litoral del Paraná, constituido por barcos de doble arrastre demersal de camarón, y aquel que potencialmente tendría su pesca más perjudicada con el proyecto. Aun así, los pescadores de este municipio aceptaron la instalación de una pequeña cantidad de arrecifes artificiales en tres puntos es- 
pecíficos en los que arrecifes rocosos ya estaban naturalmente, con la intención de estimular algunas pesquerías de peces (notablemente, ninguna de ellas en frente a su municipio). Inversamente, la propuesta más osada, que preveía la instalación de unidades antiarrastre, fue formulada por el municipio de Guaraqueçaba, que concentra los pescadores más pobres y desamparados, practicando una pesca de menor porte. Pero es preciso considerar que los pesqueros de este municipio, en frente a la isla de Superagüí, constituyen el área más productiva del litoral del Estado, atrayendo las mayores presiones de pesca, tanto de la flota local cuanto de aquella basada en Estados vecinos.

Las sugerencias y preocupaciones de los pescadores enfatizaron tanto los potenciales efectos positivos como los riesgos que las estructuras propuestas causarían a las diversas prácticas pesqueras. Se confirmó el postulado original del proyecto de que la disposición adecuada de los alineamientos de arrecifes artificiales y unidades antiarrastre podría permitir el arrastre local de pequeña escala al mismo tiempo en que cohibiría el arrastre de mayor porte (de puertas o parejas), bien como otras pescas industriales. Los pescadores confirmaron ampliamente las ventajas de ese arreglo para la pesca de pequeña escala, en la forma de mayores capturas y, tal vez más importante, por la protección dada a las redes de enmalle de pescado contra el atropellamiento y destrucción por las embarcaciones de gran porte, notablemente los arrastreros de pareja.

Sin embargo, el proceso reveló lagunas críticas en el conocimiento de los investigadores sobre la pesca y la ecología locales, y sobre los posibles impactos del proyecto sobre éstas. En particular, existía la expectativa de que las prácticas con red de enmalle, particularmente las fijas, no serían perjudicadas. A pesar del gran tamaño de estas redes (algunas traspasando los $2 \mathrm{~km}$ de extensión), los investigadores creían que, siendo las posiciones de los arrecifes conocidas, los pescadores podrían adaptar sus prácticas para evitarlos. $\mathrm{Al}$ contrario, muchos pescadores se mostraron bastante recelosos de que las estructuras acabasen por aprisionar también las redes de enmalle, en el caso de condiciones meteoceanográficas adversas. Sin embargo, por lo menos en el caso de las redes a la deriva vigiladas, algunos pescadores afirmaron que el control sería posible.

De cualquier forma, las propuestas se caracterizaron por una demanda mayor por los arrecifes artificiales, y un uso parsimonioso de las unidades antiarrastre. En el caso de los primeros, los pescadores entendieron que sería más fácil la recuperación de redes de enmalle en caso de aprisionamiento. Con la misma intención, los pescadores propusieron que el diseño de las unidades 
antiarrastre fuera modificado para que los rieles de hierro utilizados fueran paralelos y no perpendiculares. Evidentemente, las estructuras perderían eficacia con esa modificación, mas la lógica subyacente es la de que, como la red de arrastre penetra algunos centímetros en el sustrato de fondo, sería de cualquier modo retenida por la estructura. Ya los otros tipos de red no serían afectados. Vale notar que los pescadores acataron la solicitud de los investigadores, hecha al comienzo de cada reunión, de no alterar el diseño de las estructuras individuales, salvo su número y distribución, aceptando el argumento de que hay prácticamente infinitas posibilidades en términos de forma, y de que, en ese sentido, era aceptable confiar en la pericia científica.

Finalmente, fue unánime entre los pescadores la idea de que los arrecifes artificiales podrían ser lanzados también sobre substrato duro, para incrementar el hábitat rocoso de los arrecifes naturales existentes y, correspondientemente, su producción pesquera. La proposición tiene sentido, una vez que tales formas naturales en el litoral del Paraná tienen pequeñas dimensiones y son relativamente planas o poco accidentadas, destacándose poco por arriba del substrato arenoso. Así, los arrecifes artificiales podrían aumentar el volumen y complejidad topológica de estos ambientes.

\section{El proceso político e institucional}

Desde el punto de vista técnico, de evaluación de los posibles impactos del proyecto sobre los pescadores, y de obtención de su contribución para el diseño de la intervención, se puede argumentar que la participación fue suficiente (véase tabla 2).

Desde el punto de vista jurídico, la participación fue suficiente para los propósitos del licenciamiento ambiental del proyecto. La legislación brasilera exigiría apenas una audiencia pública, proceso que sufre de algunas restricciones importantes: I) los participantes son convocados apenas de una manera genérica; 2) la información previa del proyecto es, típicamente, de difícil obtención y comprensión; y 3) las personas resuelven dudas y manifiestan sus opiniones, sin que eso constituya una instancia de modificación o redefinición del proyecto. Así, el proceso aquí relatado fue mucho más allá de lo exigido.

Ya desde una perspectiva sociopolítica, el nivel de participación, en términos de cantidad de personas presentes, puede parecer desalentador, considerando el gran esfuerzo realizado para garantizar la presencia física de los pescadores. Por otro lado, es preciso considerar que desde 1996 ha habido 
procesos de implantación de arrecifes artificiales en la misma área, donde los pescadores fueron consultados a través de encuestas y reuniones, de forma que cuando esta consulta tuvo lugar el tema no era nuevo para este público y ya podía existir alguna maduración de la discusión. La baja participación parece entonces reflejar factores de tipo político, como el bajo grado de conciencia y activismo previos en la población objetivo.

Evaluar el alcance cuantitativo de la participación obtenida en esta consulta debe partir de considerar que los pescadores son una categoría fraccionada económica, geográfica y culturalmente, además de poco organizada, con dificultades para defender sus intereses colectivos, y, en gran parte, sometida a formas de dominación paternalistas y clientelistas. También se deben considerar los antecedentes locales, pues muchas veces diferentes agentes, sean investigadores, ONG, o el poder público, tomaron decisiones que afectan a los pescadores sin consultarlos o consultando apenas a los líderes formales. Y cuando, excepcionalmente, hubo una convocatoria mayor, ésta no se hizo con la amplitud, tiempo e información previa suficientes para poderse afirmar que no participar fue decisión del convocado, como en este caso. Bajo esta óptica, la participación en este proceso de consulta pública puede ser considerada significativa, sea por el número de pescadores presentes como por su representatividad municipal y de tipos de pesca e intereses.

Desde el punto de vista cualitativo, el proceso permitió y favoreció la formación de opinión individual y colectiva, dando la información, el tiempo, los elementos y los procedimientos necesarios, así como la garantía de expresión de todos, reforzando la voz de los más débiles. En ese sentido, el proceso fue altamente democrático y un ejemplo de negociación entre subsectores con diferentes intereses, donde los líderes formales y los más poderosos económicamente no pudieron imponer sus puntos de vista de la forma habitual en que eso sucede. Esto no quiere decir que las desigualdades y las relaciones de poder no hayan operado, pero seguramente lo hicieron en menor medida que lo habitual.

\section{Conclusiones y recomendaciones}

Se interpreta, pues, que se produjo un proceso de consulta verdaderamente democrático y participativo, sin precedentes en la historia de la gestión de la pesca en el Paraná, que creó condiciones concretas y legítimas para la propuesta de modificaciones en el proyecto por los diversos grupos de interés existentes entre los pescadores. 
Este hecho, si así fuera comprendido y valorado socialmente, debería inaugurar una nueva fase en la gestión pesquera de la región, en la que no fuera más posible tomar decisiones o implantar medidas que afecten de forma importante las condiciones de la pesca y de la población que de ella depende, sin una consulta previa a los afectados que tenga una calidad democrática igual o todavía superior a ésta. Para eso es fundamental divulgar y reflexionar sobre esta experiencia. Cada parte involucrada en la gestión pesquera debe repensar su lugar y procedimientos, comenzando por los técnicos que conciben las medidas y las instituciones ordenadoras que acostumbran a actuar como si la mera razón técnica pudiese resolver todos los problemas sociales, debiendo entonces ser impuesta a pesar de los impactos sociales negativos y de la eventual voluntad opuesta de los afectados. Pero, más importante que eso, es que los propios pescadores se apropien de esta experiencia como una referencia, de forma de comenzar a practicar formas de reunirse, discutir, madurar la comprensión de las cosas y tomar decisiones que expresen más cabalmente los intereses colectivos. Y que, frente a una medida que pueda afectar los recursos o condiciones de la pesca, sean capaces de exigir procedimientos de discusión donde no sólo sean oídos, sino que participen de las decisiones.

Es fundamental comprender que la más amplia y calificada participación social en las tomas de decisión no es solamente un requisito democrático, sino que resulta clave para la eficacia de la gestión, porque es la única forma de comprometer a los sectores involucrados para que, junto al poder estatal, sean corresponsables por las propuestas, y por su ejecución, monitoreo y evaluación.

\section{Referencias}

Andriguetto-Filho, J. M. (I999). Sistemas técnicos de Pesca e suas Dinâmicas de Transformação no litoral do Paraná, Brasil. Tese (Doutorado em Meio Ambiente e Desenvolvimento), Universidade Federal do Paraná (UFPR) e Université de Bordeaux II, Curitiba, 254 pp + anexos.

Andriguetto-Filho, J. M., Chaves, P. T., Santos, C. y Liberati, S. A. (2006a). Diagnóstico da pesca no litoral do estado do Paraná. En Isaac, V. J. (org), Martins, A. S., Haimovici, M., Andriguetto-Filho, J. M., A pesca marinha e estuarina do Brasil no início do século XXI: recursos, tecnologias, aspectos socioeconômicos e institucionais (I I 7-I 40). Projeto Recos: Uso e Apropriação dos Recursos Costeiros. Grupo Temático: Modelo Gerencial da pesca. Belém: Universidade Federal do Pará. 
Andriguetto-Filho, J. M., Pierri, N. E., Krul, R., Natividade, C. D., FeiTOSA, S. (2006b). Relatório final do componente de participação pública. Participação dos Pescadores no planejamento do Projeto: Paraná Tecnologia, Recuperação da biodiversidade marinha e dos estoques pesqueiros na plataforma rasa do Estado do Paraná através da instalação de Recifes Artificiais de Recrutamento Larval (RRL) e de um Sistema Anti-Arrasto (SAA). Instituto de Pesquisa e Conservação da Natureza, Idéia Ambiental. Curitiba.

Bigarella, J. J., Becker, R. D., Matos, D. J., Werner, A. (I978). A Serra do Mar e a porção oriental do Estado do Paraná. Secretaria de Estado do Planejamento do Paraná.

Brandini, F. (2003). Relatório final do projeto recifes artificiais marinhos: uma proposta de conservação da biodiversidade e desenvolvimento da pesca artesanal através da criação de um «Parque» Marinho na Costa do Estado do Paraná. [s.l.]. Ministério da Ciência e Tecnologia (РADCt III/CIAMB). Dias Neto, J., Dornelles, L. D. C. (I996). Diagnóstico da pesca maritima do Brasil. Coleção Meio Ambiente. Série Estudos Pesca, 20. Brasília: IBAma.

FunPar (2005). Elaboração do diagnóstico ambiental para o Licenciamento Ambiental de recifes artificiais com finalidade de proteção, recuperação da biota e pesquisa. Curitiba: Universidade Federal do Paraná.

IBGE, Instituto Brasileiro de Geografia e Estatística. (2007). Contagem Populacional. Río de Janeiro. Disponible en <http://www.ibge.gov.br>.

Natividade, C. D., Pereira, M. J. C. F., Andriguetto, J. M. (2004). Smallscale Fishing Landings on the Coast of the State of Paraná, Brazil from 1975 to 2000, with Emphasis on Shrimp Data. Journal of Coastal Research, 39 (Proceedings of the 8th International Coastal Symposium).

Pierri, N., Angulo, R. J., Souza, M. C., Kim, M. K. (2006). A ocupação do solo no litoral paranaense: condicionantes, conflitos e tendências. Desenvolvimento e Meio Ambiente, I3: I37-I67.

SAntos, G. C. dos. (2007). A utilização dos recifes artificiais e unidades antiarrasto como ferramenta da gestão pesqueira: histórico e percepção dos principais atores envolvidos. Monografia de Graduação. Curso de Graduação em Oceanografia. Centro de Estudos do Mar. Universidade Federal do Paraná. SEMA, Secretaria de Estado de Meio Ambiente e Recursos Hídricos. (2006). Paraná: Mar e Costa. Subsídios ao Ordenamento das Áreas Estuarina e Costeira do Paraná: Projeto Gestão Integrada da Zona Costeira do Paraná com ênfase na Área Marinha. Programa Nacional de Meio Ambiente, PNMA II. 
Vasconcellos, M., Diegues, A. C., Sales, R. R. de. (2005). Relatório Integrado: Diagnóstico da pesca artesanal no Brasil como subsídio para o fortalecimento institucional da Secretaria Especial de Aqüicultura e Pesca. Versão preliminar. Relatório técnico.

\section{Sobre los autores}

José Milton AndriguetTo-Filho es oceanógrafo de la Universidad Federal de Paraná, Programa de Pos-graduación en Medio Ambiente y Desarrollo. Su correo electrónico es: <jmandri@ufpr.br>. NAína Pierri es socióloga del Programa de Pos-graduación en Medio Ambiente y Desarrollo de la Universidad Federal de Paraná. Su correo electrónico es <naina@cem.ufpr.br>. 
